\title{
A COUNTER-EXAMPLE BY YAGITA
}

\author{
NIKITA A. KARPENKO
}

\begin{abstract}
According to a 2018 preprint by Nobuaki Yagita, the conjecture on a relationship between $K$ - and Chow theories for a generically twisted flag variety under a split semisimple algebraic group $G$, due to the author, fails for $G$ the spinor group $\operatorname{Spin}(17)$. Yagita's tools include a Brown-Peterson version of algebraic cobordism, ordinary and connective Morava $K$-theories, as well as Grothendieck motives related to various cohomology theories over fields of characteristic 0 . We provide a proof using only the $K$ and Chow theories themselves and extend the (slightly modified) example to arbitrary characteristic.
\end{abstract}

\section{Contents}

1. Introduction 1

2. Basic notation 3

3. Steenrod operations 3

4. "Connective $K$-theory" $\quad 5$

References

\section{INTRODUCTION}

For a smooth algebraic variety $X$ over an arbitrary field $F$, we write $\mathrm{CH}(X)$ for the Chow ring of $X$ and $K(X)$ for the Grothendieck ring of vector bundles on $X$. There is a canonical surjective homomorphism of graded rings $\varphi_{X}: \mathrm{CH}(X) \rightarrow$ Chow $K(X)$ onto the ring associated with the Chow filtration on $K(X),[12, \S 3]$. The elements in the kernel of $\varphi_{X}$ are known to be of finite order.

Let $G$ be a split semisimple algebraic group over $F$ and let $X$ be the variety of its Borel subgroups, twisted by a generic $G$-torsor. It has been conjectured in $[8$, Conjecture 1.1] that $\varphi_{X}$ is an isomorphism. This conjecture appears already in $[9, \S 1]$ in form of a question. The answer depends only on $G$ and does not depend on the choice of a generic $G$-torsor. For any given $G$, the variety of Borel subgroups can be replaced by the variety of special (i.e., not admitting non-trivial torsors over fields) parabolic subgroups of any given type: an equivalent version of the conjecture/question is produced this way.

Date: 22 May 2019.

Key words and phrases. Algebraic groups; generic torsors; projective homogeneous varieties; Chow groups. Mathematical Subject Classification (2010): 20G15; 14C25.

This work has been accomplished during author's stay at the Max-Planck Institute for Mathematics in Bonn. 
For any odd integer $l \geq 1$, the cases $G=\operatorname{Spin}(l)$ and $G=\operatorname{Spin}(l+1)$ of this conjecture are equivalent and mean that $\varphi_{X}$ is an isomorphism for $X$ the highest orthogonal grassmannian of a generic $l$-dimensional quadratic form of trivial Clifford invariant. The index $\operatorname{ind}(X)$ (= the greatest common divisor of the degrees of closed points on $X$ ) has been computed for such $X$ by Burt Totaro in [18]. In particular, one has $\operatorname{ind}(X)=2^{4}$ in the case of $l=17$.

According to Nobuaki Yagita the above conjecture fails for $G=\operatorname{Spin}(17)$. The following statement appears in [21] over a field of characteristic 0 and is extended here to arbitrary characteristic:

Theorem 1.1 (cf. [21]). Let $X$ be the highest orthogonal grassmannian of an arbitrary non-degenerate 17-dimensional quadratic form over $F$ of trivial Clifford invariant. Assume that ind $X=2^{4}$. Then $\varphi_{X}$ is not an isomorphism.

To provide more details, let us write $c_{k}^{\mathrm{CH}} \in \mathrm{CH}^{k}(X), k=1, \ldots, 8$, for the Chern classes of the dual to the (rank-8) tautological vector bundle on $X$. Since the Clifford invariant of the quadratic form is trivial, the group $\mathrm{CH}^{1}(X)$ is generated by an element $e^{\mathrm{CH}}$ satisfying the relation $2 e^{\mathrm{CH}}=c_{1}^{\mathrm{CH}}$. By Corollary 3.4 and Proposition 4.4, proved below, the product

$$
c_{2}^{\mathrm{CH}} c_{3}^{\mathrm{CH}} c_{6}^{\mathrm{CH}} c_{7}^{\mathrm{CH}}\left(e^{\mathrm{CH}}\right)^{15} \in \mathrm{CH}^{33}(X)
$$

is not divisible by 2 , but its image under $\varphi_{X}$ is.

The proof of the non-divisibility is given in $\S 3$ and makes use of the Steenrod operations on $\mathrm{Ch}(X):=\mathrm{CH}(X) / 2 \mathrm{CH}(X)$. Cohomological operations in algebraic geometry have been introduced by Vladimir Voevodsky in the context of motivic cohomology, [20]. The Steenrod operations on the Chow groups modulo a prime $p$ have been constructed over fields of characteristic $\neq p$ by Patrick Brosnan in [1]. Very recently, they've been constructed over fields of characteristic $p$ by Eric Primozic, [16] (see also [17]).

The computations in $\S 3$ are quite mysterious. Not only because the Steenrod operations themselves are so, but also because we do not know much about $\mathrm{CH}(X)$. Still it is possible to determine the values of the operations on certain "easy" part of $\mathrm{CH}(X)$ : the subring generated by the Chern classes of the tautological vector bundle on $X$. This subring is explicitly known and very rigid: it does not change under extensions of the base field (see, e.g., [11, Theorem 2.1]). On the other hand, when taken modulo 2, it vanishes over an algebraic closure. We finish by computing over an algebraic closure, where the whole integral Chow ring is known. But instead of computing modulo 2, we work modulo a higher 2-power, despite of the fact that the operations themselves are only defined modulo $2 \ldots$

The proof of the divisibility is given in $\S 4$ and makes use of an imitation $C K(X)$ of the connective $K$-theory of $X$, built from the terms of the Chow filtration on $K(X)$. One could expect this part to be a boring computation because $K(X)$ is computable as a ring. In the generic case (as in the conjecture), which is the only case we are really interested in, the Chow filtration on $K(X)$ is also computable. However, making a direct computation turns out to be extremely complicated and has been not worked out. The idea of the computation actually made is borrowed from [21]. It looks like it only uses the structure of the modulo-2 Chow ring of $\bar{X}$ (which is $X$ over an algebraic closure of the base field). This structure has been beautifully described by Alexander Vishik in [19] (see also [4, 
$\S 86])$. It is simpler than that of the integral Chow ring $\mathrm{CH}(\bar{X})$ and much more simpler than that of $K(X)=K(\bar{X})$.

Our proofs, at the same time, are guided by and differ in many ways from [21], where, e.g., a Brown-Peterson version of algebraic cobordism, ordinary and connective Morava $K$-theories, as well as Grothendieck motives related to various theories are involved, in the arguments as well as in the statements. None of these appear here. Also it seems, some arguments in [21] need justification (or modification). For instance, multiplication in various additive groups, related to motives, is constantly used there.

Let us conclude by remark that the conjecture we are discussing has been verified for many groups including the spinor ones up to $\operatorname{Spin}(12)$, see [10]. The cases of Spin(13/14) and $\operatorname{Spin}(15 / 16)$ are still open.

\section{BASIC NOTATION}

Throughout this note, $n$ is an integer $\geq 1, q$ is a non-degenerate quadratic form of dimension $2 n+1$ over a field $F$, and $X$ is the highest orthogonal grassmannian of $q$, i.e., its variety of $n$-dimensional totally isotropic subspaces.

For every integer $k$, let $\mathrm{CH}^{k}(X)$ be the codimension- $k$ component of the graded Chow ring $\mathrm{CH}(X)$. (On some rare occasions we write $\mathrm{CH}_{k}(X)$ for the dimension- $k$ component $\mathrm{CH}^{\operatorname{dim} X-k}(X)$.) For $k \geq 0$, let $c_{k}^{\mathrm{CH}} \in \mathrm{CH}^{k}(X)$ be the $k$ th Chern class of the dual to the (rank- $n$ ) tautological vector bundle $\mathcal{T}$ on $X$. In particular, $c_{0}^{\mathrm{CH}}=1, c_{k}^{\mathrm{CH}}=0$ for $k>n$, and $(-1)^{k} c_{k}^{\mathrm{CH}}$ is the $k$ th Chern class of $\mathcal{T}$ for any $k$. Note that by [4, Proposition 86.13], $c_{k}^{\mathrm{CH}}$ itself (without the sign correction) is the $k$ th Segre class of $\mathcal{T}$.

We also consider the Chow ring $\mathrm{Ch}(X):=\mathrm{CH}(X) / 2 \mathrm{CH}(X)$ with coefficients in $\mathbb{F}_{2}:=$ $\mathbb{Z} / 2 \mathbb{Z}$ and write $c_{k}$ for the class in $\mathrm{Ch}^{k}(X)$ of the element $c_{k}^{\mathrm{CH}} \in \mathrm{CH}^{k}(X)$.

Remaining notation will be introduced on the go.

\section{Steenrod operations}

We consider the cohomological Steenrod operation $S_{X}: \operatorname{Ch}(X) \rightarrow \operatorname{Ch}(X)$, as in $[4$, Chapter XI]. We do not exclude the characteristic 2 (excluded in [4, Chapter XI]), where the reference is [16]. All the properties of $S_{X}$ we need are available in arbitrary characteristic. For characteristic 2 they are obtained in Corollary 2.4 (commutation with pull-backs), Proposition 5.1 (Cartan formula), Corollary 6.3 (relation with squaring), Proposition 6.4 (vanishing), and Proposition 7.1 (relation with push-forwards) of [16].

For any $i \geq 0$, we write $S_{X}^{i}$ for the $i$ th homogeneous component $S_{X}^{i}: \operatorname{Ch}(X) \rightarrow \mathrm{Ch}^{+i}(X)$ of the total operation $S_{X}$.

Proposition 3.1. For any $k \geq 0$ and any $i \geq 0$, one has

$$
S_{X}^{i}\left(c_{k}\right)=\left(\begin{array}{c}
k-1 \\
i
\end{array}\right) c_{k+i} .
$$

Proof. We adapt the proof of $[4$, Theorem 89.1]. The formula $[4,(86.5)]$ is replaced by

$$
c_{k}=\left(s_{*} \circ t^{*}\right)\left(h^{n-1+k}\right),
$$

where $h \in \mathrm{Ch}^{1}\left(X_{1}\right)$ is the class of a hyperplane section of the quadric $X_{1}$ of $q$, and $s_{*}, t^{*}$ are as in $[4,86.5]$ : for $X_{n}:=X, t$ is the projection of the flag variety $X_{1 \subset n} \subset X_{1} \times X_{n}$ 
onto $X_{1}$ and $s$ is the other projection $X_{1 \subset n} \rightarrow X_{n}$. Since $s$ it the projective bundle given by the tautological vector bundle on $X_{n}$ and $h$ is the Euler class of the canonical line bundle on $X_{1}$, formula (3.2) follows from the definition of Segre classes as in [5, Chapter $3]$ or $[4, \S 58 . \mathrm{A}]$.

Instead of the formula for the value of the Steenrod operation on the elements in $\mathrm{Ch}\left(X_{1}\right)$ which appear in the proof of [4, Theorem 89.1], we apply the formula

$$
S_{X_{1}}\left(h^{k}\right)=(1+h)^{k} h^{k}
$$

which holds for any $k \geq 0$ as a consequence of the formula

$$
S_{X_{1}}(h)=S_{X_{1}}^{0}(h)+S_{X_{1}}^{1}(h)=h+h^{2}
$$

(for characteristic $\neq 2$ see [4, Theorem 61.13 and Example 61.16]) and Cartan formula $([4$, Corollary 61.15] for characteristic $\neq 2)$.

We now specify the situation we are considering. First we assume that the Clifford invariant of the quadratic form $q$ is trivial. This implies that $c_{1}^{\mathrm{CH}}=2 e^{\mathrm{CH}}$ for some $e^{\mathrm{CH}} \in \mathrm{CH}^{1}(X)$ (see, e.g., [4, Exercise 88.14(1)]). We write $e \in \mathrm{Ch}^{1}(X)$ for the modulo-2 class of $e^{\mathrm{CH}}$.

Then we additionally assume that $n=8$ and the index of $X$ is 16 . In this case we have a homomorphism (deg /16): $\mathrm{Ch}(X) \rightarrow \mathbb{F}_{2}$ mapping the class of any closed point on $X$ to the class modulo 2 of its degree divided by 16 (and mapping to 0 the classes of positive dimensions).

Proposition 3.3. $(\mathrm{deg} / 16)\left(S_{X}^{3}\left(c_{2} c_{3} c_{6} c_{7} e^{15}\right)\right) \neq 0$.

Proof. By Proposition 3.1 we have: $S_{X}\left(c_{2}\right)=c_{2}+c_{3}, S_{X}\left(c_{3}\right)=c_{3}+c_{5}, S_{X}\left(c_{6}\right)=c_{6}+c_{7}$, and $S_{X}\left(c_{7}\right)=c_{7}$. Besides, $S_{X}(e)=e+e^{2}$. It follows that

$$
\begin{aligned}
S_{X}\left(c_{2} c_{3} c_{6} c_{7} e^{15}\right)=S_{X}\left(c_{2}\right) S_{X}\left(c_{3}\right) S_{X}\left(c_{6}\right) S_{X}\left(c_{7}\right) S_{X}(e)^{15} & = \\
& \left(c_{2}+c_{3}\right)\left(c_{3}+c_{5}\right)\left(c_{6}+c_{7}\right) c_{7}\left(e+e^{2}\right)^{15} .
\end{aligned}
$$

Let $\bar{X}$ be $X$ over an algebraic closure of $F$. The ring $\mathrm{CH}(\bar{X})$ has been computed (for arbitrary $n$ ) by Alexander Vishik in [19] (see also $[4, \S 86]$ ). The group $\mathrm{CH}(\bar{X})$ is free of finite rank. For any $k>0$, the image of $c_{k}^{\mathrm{CH}}$ in $\mathrm{CH}(\bar{X})$ is $2 e_{k}^{\mathrm{CH}}$ for certain $e_{k}^{\mathrm{CH}} \in \mathrm{CH}^{k}(\bar{X})$. (In particular, $e_{1}^{\mathrm{CH}}$ is the image of $e^{\mathrm{CH}}$.) The products $e_{J}^{\mathrm{CH}}:=\prod_{k \in J} e_{k}^{\mathrm{CH}}$, where $J$ runs over the subsets of $\{1, \ldots, n\}$, form an additive basis of $\mathrm{CH}(\bar{X})$. In particular, the product $e_{1}^{\mathrm{CH}} e_{2}^{\mathrm{CH}} \ldots e_{n}^{\mathrm{CH}} \in \mathrm{CH}^{\operatorname{dim} X}(\bar{X})=\mathrm{CH}_{0}(\bar{X})$ is the class of a rational point. Note that $\operatorname{dim} X=n(n+1) / 2$. In our special case of $n=8$ we have $\operatorname{dim} X=36$.

The product

$$
\left(c_{2}^{\mathrm{CH}}+c_{3}^{\mathrm{CH}}\right)\left(c_{3}^{\mathrm{CH}}+c_{5}^{\mathrm{CH}}\right)\left(c_{6}^{\mathrm{CH}}+c_{7}^{\mathrm{CH}}\right) c_{7}^{\mathrm{CH}}\left(e^{\mathrm{CH}}+\left(e^{\mathrm{CH}}\right)^{2}\right)^{15}
$$

is an integral representative of $S_{X}\left(c_{2} c_{3} c_{6} c_{7} e^{15}\right)$. The degree-36 part of this product is an integral representative of $S_{X}^{3}\left(c_{2} c_{3} c_{6} c_{7} e^{15}\right)$. This degree-36 part is a sum, where each summand is a product of 4 elements $c_{k}^{\mathrm{CH}}$ (with various $k$ ) and a power of $e^{\mathrm{CH}}$. One of them is

$$
c_{3}^{\mathrm{CH}} c_{5}^{\mathrm{CH}} c_{6}^{\mathrm{CH}} c_{7}^{\mathrm{CH}}\left(e^{\mathrm{CH}}\right)^{15},
$$


an integral representative of $S^{1}\left(c_{2}\right) S^{2}\left(c_{3}\right) c_{6} c_{7} e^{15}$, which is an element of degree 16 modulo 32. Indeed, the image in $\mathrm{CH}(\bar{X})$ of $c_{3}^{\mathrm{CH}} c_{5}^{\mathrm{CH}} c_{6}^{\mathrm{CH}} c_{7}^{\mathrm{CH}}$ is $16 e_{3}^{\mathrm{CH}} e_{5}^{\mathrm{CH}} e_{6}^{\mathrm{CH}} e_{7}^{\mathrm{CH}}$ and the image of $\left(e^{\mathrm{CH}}\right)^{15}$ is $\left(e_{1}^{\mathrm{CH}}\right)^{15}=e_{1}^{\mathrm{CH}}\left(e_{1}^{\mathrm{CH}}\right)^{2}\left(e_{1}^{\mathrm{CH}}\right)^{4}\left(e_{1}^{\mathrm{CH}}\right)^{8}$ which is congruent modulo 2 to $e_{1}^{\mathrm{CH}} e_{2}^{\mathrm{CH}} e_{4}^{\mathrm{CH}} e_{8}^{\mathrm{CH}}$ because of the modulo-2 relation $\left(e_{k}^{\mathrm{CH}}\right)^{2} \equiv e_{2 k}^{\mathrm{CH}}$ (which holds for all $n$ and all $k$ ).

We claim that the degree of every other summand in the degree-36 part is divisible by 32. Indeed, since $\left(e_{1}^{\mathrm{CH}}\right)^{16}$ is 0 modulo 2 , the degree of every summand containing a power of $e^{\mathrm{CH}}$ greater than 15 is divisible by 32 . The remaining summand is $c_{2}^{\mathrm{CH}} c_{5}^{\mathrm{CH}} c_{7}^{\mathrm{CH}} c_{7}^{\mathrm{CH}}\left(e^{\mathrm{CH}}\right)^{15}$. Because of the formula

$$
\left(c_{k}^{\mathrm{CH}}\right)^{2}=2\left(c_{k-1}^{\mathrm{CH}} c_{k+1}^{\mathrm{CH}}-c_{k-2}^{\mathrm{CH}} c_{k+2}^{\mathrm{CH}}+\cdots-(-1)^{k} c_{0}^{\mathrm{CH}} c_{2 k}^{\mathrm{CH}}\right) \in \mathrm{CH}(X)
$$

(which holds again for all $n$ and $k$, but this time over $F$, see $[4, \S 86]$ or $[11$, Theorem 2.1]) its image in $\mathrm{CH}(\bar{X})$ is divisible by 32 .

Corollary 3.4. Let $X$ be the highest orthogonal grassmannian of a non-degenerate 17 dimensional quadratic form of trivial Clifford invariant over an arbitrary field. Assume that $\operatorname{ind}(X)=16$. Then the element $c_{2}^{\mathrm{CH}} c_{3}^{\mathrm{CH}} c_{6}^{\mathrm{CH}} c_{7}^{\mathrm{CH}}\left(e^{\mathrm{CH}}\right)^{15} \in \mathrm{CH}^{33}(X)$ is not divisible by 2 .

\section{4. "COnNECTIVE $K$-THEORY"}

In this section we work with the Grothendieck ring $K(X)$. By [13], $K(X)$ is identified with a subring in $K(\bar{X})$ (where $X$ is as in $\S 2$; we do not assume yet that the Clifford invariant of $q$ is trivial). We consider the Chow filtration on $K(X)$ and write $K(X)^{(i)}$ for its $i$ th term. The index $i$ is allowed to be any integer: $K(X)^{(i)}=K(X)^{(0)}=K(X)$ for negative $i$.

Note that $K(X)^{(i)} \subset K(\bar{X})^{(i)}$ for any $i$.

For $k \geq 0$, we write $c_{k}^{K} \in K(X)^{(k)}$ for the $K$-theoretic Chern class of the tautological vector bundle on $X$, defined as in [12, Example 2.3]. For $k \geq 1$, we also define the element $e_{k}^{K} \in K(\bar{X})^{(k)}$ as $e_{k}^{K}:=0$ for $k>n$ and $e_{k}^{K}:=\left(s_{*} \circ t^{*}\right)\left(l_{n-k}^{K}\right)$ for $k \leq n$, where $s, t$ are as in the proof of Proposition 3.1 and $l_{n-k}^{K} \in K\left(\bar{X}_{1}\right)^{n+k-1}$ is the class of a linear projective $(n-k)$-subspace on the quadric $X_{1}$. (Note that $\operatorname{dim} X_{1}=\operatorname{dim} q-2=2 n-1$.)

Lemma 4.1. For any $k \geq 1$, the element $2 e_{k}^{K}+e_{k+1}^{K}$ of $K(\bar{X})^{(k)}$ actually belongs to $K(X)^{(k)}$ and is congruent modulo $K(X)^{(k+1)}$ to $c_{k}^{K}$. More precisely, the difference

$$
\left(2 e_{k}^{K}+e_{k+1}^{K}\right)-c_{k}^{K}
$$

is a sum of monomials in $c_{1}^{K}, \ldots, c_{n}^{K}$ of degree greater than $k$ (where for any $r \geq 0$, the degree of $c_{r}^{K}$ is defined as $\left.r\right)$.

Proof. Let $h^{K} \in K\left(X_{1}\right)^{(1)}$ be the class of a linear section of the quadric $X_{1}$. By [7, §3] (see $[6, \S 2]$ for more details), $\left(h^{K}\right)^{n+k-1}=2 l_{n-k}^{K}+l_{n-k-1}^{K} \in K\left(\bar{X}_{1}\right)$ for any $k \geq 1$. Therefore, to prove Lemma 4.1 is to prove that $\left(s_{*} \circ t^{*}\right)\left(\left(h^{K}\right)^{n+k-1}\right)$ equals $c_{k}^{K}$ plus a sum of monomials in $c_{1}^{K}, \ldots, c_{n}^{K}$ of degree greater than $k$.

Since the modulo-2 formula (3.2) holds integrally, the element $\left(s_{*} \circ t^{*}\right)\left(\left(h^{K}\right)^{n+k-1}\right)$ is $c_{k}^{K}$ modulo $K(X)^{(k+1)}$.

To prove the more precise statement of Lemma 4.1, let us consider the generic $(2 n+1)$ dimensional quadratic form $q^{\text {gen }}$ as in $[11, \S 3]: q^{\text {gen }}$ is the quadratic form over the field $F^{\text {gen }}$ 
of rational functions over $F$ in $n(n+1) / 2$ variables, whose coefficients are the variables. For the maximal orthogonal grassmannian $X^{\text {gen }}$ of $q^{\text {gen }}$, since the ring $\mathrm{CH}\left(X^{\text {gen }}\right)$ is generated by $c_{1}^{\mathrm{CH}}, \ldots, c_{n}^{\mathrm{CH}}$ (see, [14] or [11, Theorem 6.1]), the group $K\left(X^{\mathrm{gen}}\right)^{(k+1)}$ is generated by monomials in $c_{1}^{K}, \ldots, c_{n}^{K}$ of degree greater than $k$. Therefore, the statement we want for $q$ holds for $q^{\text {gen }}$. Since over an algebraic closure $\bar{F}^{\text {gen }}$ of $F^{\text {gen }}$ the forms $q^{\text {gen }}$ and $q$ become isomorphic, the statement holds for $q_{\bar{F}}$ gen. Since the change of fields homomorphism $K(X) \rightarrow K\left(X_{\bar{F} \text { gen }}\right)$ is injective, the statement holds for $q$ as well.

We consider the following imitation of the connective $K$-theory of $X$ (for definition of the connective $K$-theory itself see [2] or [3]). Let $C K(X)$ be the $\mathbb{Z}$-graded ring with $C K^{i}(X):=K(X)^{(i)}$ for any $i$. We write $v \in C K^{-1}(X)$ for the element $1 \in K(X)^{(-1)}$. So, $v$ is an element of degree -1 and $C K(X)$ is the subring of the Laurent polynomial ring $K(X)\left[v, v^{-1}\right]$ consisting of the polynomials $\sum_{i} a_{i} v^{i}$ satisfying the condition $a_{i} \in K(X)^{(-i)}$ for any $i$. In particular, $a_{i}=0$ for $i<-\operatorname{dim} X$.

Example 4.2. The graded $\operatorname{ring} C K(\operatorname{Spec} F)$ is the polynomial $\operatorname{ring} \mathbb{Z}[v]$.

We write $c_{k}^{C K}$ and $e_{k}^{C K}$ for $c_{k}^{K} v^{-k} \in C K^{k}(X)$ and $e_{k}^{K} v^{-k} \in C K^{k}(\bar{X})$.

Let $I(X) \subset C K(X)$ be the ideal generated by 2 and $v$. Note that

$$
C K(X) / v C K(X)=\operatorname{Chow} K(X) \text { and } C K(X) / I=\operatorname{Chow} K(X) / 2 \operatorname{Chow} K(X) .
$$

Lemma 4.3. For any $k \geq 1$ one has: $\left(e_{k}^{C K}\right)^{2} \equiv e_{2 k}^{C K}(\bmod I(\bar{X}))$.

Proof. For any $k \geq 1$, the element $e_{k}^{C K}$ has been defined the way that its class modulo $v C K(\bar{X})$ coincides with the image of $e_{k}^{\mathrm{CH}} \in \mathrm{CH}^{k}(\bar{X})$ under the homomorphism $\mathrm{CH}(\bar{X}) \rightarrow$ $C K(\bar{X}) / v C K(\bar{X})$ (see [4, Formula (86.5)]). In particular, the class of $e_{k}^{C K}$ modulo $I(\bar{X})$ coincides with the image of $e_{k} \in \mathrm{Ch}^{k}(\bar{X})$ under the homomorphism $\mathrm{Ch}(\bar{X}) \rightarrow C K(\bar{X}) / I(\bar{X})$. The congruence follows from the relation $e_{k}^{2}=e_{2 k}$ we have in $\operatorname{Ch}(\bar{X})$.

Below we are assuming that the Clifford invariant of the quadratic form $q$ is trivial. Therefore the subring $K(X)$ of $K(\bar{X})$ coincides with $K(\bar{X})$. The Chow filtrations on $K(X)$ and $K(\bar{X})$ may still differ. However $K(X)^{(1)}=K(\bar{X})^{(1)}$. In particular, $e^{K}:=$ $e_{1}^{K} \in K(X)^{(1)}$.

Proposition 4.4. For $n=8(\operatorname{dim} q=17)$, assume that the Clifford invariant of $q$ is trivial. Then the class of the element $c_{2}^{K} c_{3}^{K} c_{6}^{K} c_{7}^{K}\left(e^{K}\right)^{15} \in K(X)^{(33)}$ in the quotient Chow $^{33} K(X)=K(X)^{(33)} / K(X)^{(34)}$ is divisible by 2 .

Proof. We do our computations in $C K(X)$. To simplify notation, we write $c_{k}, e_{k}, e$ instead of $c_{k}^{C K}, e_{k}^{C K}, e_{1}^{C K}$ in this proof.

According to Lemma 4.1, the class of the product $c_{2} c_{3} c_{6} c_{7} e^{15} \in C K^{33}(X)$ modulo $v C K^{34}(X)$ is represented by the element

$$
x:=\left(2 e_{2}+v e_{3}\right)\left(2 e_{3}+v e_{4}\right)\left(2 e_{6}+v e_{7}\right)\left(2 e_{7}+v e_{8}\right) e^{15} \in C K^{33}(X) .
$$

All we need to prove is the inclusion $x \in I(X)$, i.e.,

$$
x \in 2 C K^{33}(X)+v C K^{34}(X) .
$$


Let us consider $x$ as an element of $C K(\bar{X})$. Obviously, $x \in I(\bar{X})^{4}$. As a first step, we claim and check that

$$
x \equiv 0 \quad\left(\bmod I(\bar{X})^{5}\right) .
$$

Indeed, the expression defining $x$ is a polynomial in $v$. Its free term is $2^{4} e_{2} e_{3} e_{6} e_{7} e^{15}$, which is 0 modulo $I(\bar{X})^{5}$ because $e_{2} \equiv e^{2}$ and $e^{16} \equiv 0$ modulo $I(\bar{X})$ by Lemma 4.3.

Concerning the coefficient to $v$ in $x$, we need to check that it is 0 modulo $I(\bar{X})^{4}$. Dividing by $2^{3}$, we get to check that

$$
\left(e_{3} e_{3} e_{6} e_{7}+e_{2} e_{4} e_{6} e_{7}+e_{2} e_{3} e_{7} e_{7}+e_{2} e_{3} e_{6} e_{8}\right) e^{15}
$$

is 0 modulo $I(\bar{X})$. The first summand in the parentheses modulo $I(\bar{X})$ is

$$
e_{3} e_{3} e_{6} e_{7} \equiv e_{6} e_{6} e_{7} \equiv 0 \text {. }
$$

Each of the remaining three summands contains $e_{k}$ with $k$ a 2 -power. Since $e_{k} \equiv e^{k}$ $(\bmod I(\bar{X}))$ for such $k$, we get 0 modulo $I(\bar{X})$ after the multiplication by $e^{15}$.

Next we check that the coefficient to $v^{2}$ in $x$ divided by $2^{2}$ is 0 modulo $I(\bar{X})$. This coefficient is a sum of 6 products multiplied by $e^{15}$. The products containing $e_{k}$ with $k$ a 2 -power are treated as before. The remaining product is $e_{3} e_{3} e_{7} e_{7}$ and is 0 modulo $I(\bar{X})$ because $e_{7}^{2}$ is so.

For the coefficients to $v^{3}$, only the argument on $e_{k}$ with $k$ a 2-power is needed. The coefficients to higher powers of $v$ are 0 by dimension reason.

We proved claim (4.6). Therefore

$$
x=2^{5} x_{0}+2^{4} v x_{1}+2^{3} v^{2} x_{2}+2^{2} v^{3} x_{3}
$$

for some $x_{k} \in C K^{33+k}(\bar{X})$ with $k=0, \ldots, 3$.

Our quadratic form $q$, as any non-degenerate 17-dimensional quadratic form of trivial Clifford invariant, completely splits over a finite field extension $L / F$ of degree dividing $2^{4}$. (This is a consequence of Albrecht Pfister's theorem on 11-dimensional quadratic forms of trivial Clifford invariant, [15].) Note that $C K\left(X_{L}\right)=C K(\bar{X})$. The composition of the change of field homomorphism $C K(X) \rightarrow C K\left(X_{L}\right)$ with the norm map $N_{L / F}: C K\left(X_{L}\right) \rightarrow$ $C K(X)$ is multiplication by the degree $[L: F]$. Since the group $K(\bar{X})$ is torsion-free and the subgroup $K(X) \subset K(\bar{X})$ is of finite index, the composition

$$
C K\left(X_{L}\right) \stackrel{N_{L / F}}{\longrightarrow} C K(X) \longrightarrow C K\left(X_{L}\right)
$$

in the other order is also multiplication by $[L: F]$. It follows that

$$
2^{4} C K(\bar{X}) \subset C K(X) \text {. }
$$

Using inclusion (4.8) we can kill $x_{0}$ and $x_{1}$ in (4.7). To deal with $x_{2}$ and $x_{3}$, however, we need some more computations.

Recall that for arbitrary $n$, the total Chow group $\mathrm{CH}(\bar{X})$ has an additive basis given by the products $e_{J}^{\mathrm{CH}}:=\prod_{k \in J} e_{k}^{\mathrm{CH}}, J \subset\{1, \ldots, n\}$. In particular, $\mathrm{CH}_{0}(\bar{X})$ and $\mathrm{CH}_{1}(\bar{X})$ are both cyclic, generated respectively by $e_{\{1, \ldots, n\}}^{\mathrm{CH}}$ and $e_{\{2, \ldots, n\}}^{\mathrm{CH}}$. For our case of $n=8$, it follows that the group $C K^{36}(\bar{X})=\mathrm{CH}^{36}(\bar{X})=\mathrm{CH}_{0}(\bar{X})$ is generated by $e_{\{1, \ldots, 8\}}:=e_{1} e_{2} \ldots e_{8}$ so that $x_{3}$ is a multiple of $e_{\{1, \ldots, 8\}}$. It also follows that the group $C K^{35}(\bar{X})$ is generated by $v e_{\{1, \ldots, 8\}}$ and $e_{\{2, \ldots, 8\}}:=e_{2} \ldots e_{8}$. Therefore, we may assume that $x_{2}$ is a multiple of $e_{\{2, \ldots, 8\}}$. 
Consider the element

$$
y:=\left(2 e_{2}+v e_{3}\right)\left(2 e_{4}+v e_{5}\right)\left(2 e_{6}+v e_{7}\right)\left(2 e_{7}+v e_{8}\right) e^{14} \in C K^{33}(X) .
$$

Computing as for (4.6), one shows that

$$
y \equiv 2^{2} v^{2} e_{\{2, \ldots, 8\}} \quad\left(\bmod I(\bar{X})^{5}\right) .
$$

It follows that vey $\equiv 2^{2} v^{3} e_{\{1, \ldots, 8\}}$ and $2 y \equiv 2^{3} v^{2} e_{\{2, \ldots, 8\}}$ modulo $I(\bar{X})^{6}$. Therefore, adding to $x$ an appropriate linear combination of vey $\in v C K^{34}(X)$ and $2 y \in 2 C K^{33}(X)$ and using (4.8), we can reduce to the case $x=0$.

Proof of Theorem 1.1. As already explained in the introduction, Theorem 1.1 follows from Corollary 3.4 and Proposition 4.4. We only need to add/recall that for any $k \geq 1$, the image of $c_{k}^{\mathrm{CH}} \in \mathrm{CH}^{k}(X)$ under the ring homomorphism $\varphi_{X}$ is represented by $c_{k}^{K} \in$ $K(X)^{(k)}$. And the image of $e^{\mathrm{CH}} \in \mathrm{CH}^{1}(X)$ is represented by $e^{K} \in K(X)^{(1)}$.

Acknowledgements. I thank Alexander Merkurjev and Nobuaki Yagita for useful comments.

\section{REFERENCES}

[1] Brosnan, P. Steenrod operations in Chow theory. Trans. Amer. Math. Soc. 355, 5 (2003), 18691903 (electronic).

[2] CAI, S. A simple description of algebraic connective $K$-theory. PhD Thesis, Los Angeles, USA, 2006, 108 pages.

[3] CAI, S. Algebraic connective K-theory and the niveau filtration. J. Pure Appl. Algebra 212, 7 (2008), $1695-1715$.

[4] Elman, R., Karpenko, N., And Merkurjev, A. The algebraic and geometric theory of quadratic forms, vol. 56 of American Mathematical Society Colloquium Publications. American Mathematical Society, Providence, RI, 2008.

[5] Fulton, W. Intersection theory, second ed., vol. 2 of Ergebnisse der Mathematik und ihrer Grenzgebiete. 3. Folge. A Series of Modern Surveys in Mathematics [Results in Mathematics and Related Areas. 3rd Series. A Series of Modern Surveys in Mathematics]. Springer-Verlag, Berlin, 1998.

[6] Karpenko, N. A. The Chow ring of a projective quadric (in Russian). PhD Thesis, Leningrad, USSR, June 1990, 82 pages. Available on author's webpage.

[7] Karpenko, N. A. Algebro-geometric invariants of quadratic forms. Algebra i Analiz 2, 1 (1990), $141-162$.

[8] Karpenko, N. A. Chow ring of generic flag varieties. Math. Nachr. 290, 16 (2017), 2641-2647.

[9] Karpenko, N. A. Chow ring of generically twisted varieties of complete flags. Adv. Math. 306 (2017), 789-806.

[10] Karpenko, N. A. On generic flag varieties of Spin(11) and Spin(12). Manuscripta Math. 157 (2018), $13-21$.

[11] Karpenko, N. A. On generic quadratic forms. Pacific J. Math. 297, 2 (2018), 367-380.

[12] Karpenko, N. A., And Merkurjev, A. S. Chow filtration on representation rings of algebraic groups. International Mathematics Research Notices, to appear.

[13] Panin, I. A. On the algebraic $K$-theory of twisted flag varieties. $K$-Theory 8, 6 (1994), 541-585.

[14] Petrov, V. A. Chow ring of generic maximal orthogonal Grassmannians. Zap. Nauchn. Sem. S.Peterburg. Otdel. Mat. Inst. Steklov. (POMI) 443 (2016), 147-150.

[15] Pfister, A. Quadratische Formen in beliebigen Körpern. Invent. Math. 1 (1966), 116-132.

[16] Primozic, E. Motivic Steenrod operations in characteristic $p$. PhD Thesis, Los Angeles, USA, 14 May 2019, 25 pages. 
[17] Primozic, E. Motivic Steenrod operations in characteristic $p$. arXiv:1903.11185 [math.AG], 26 Mar 2019, 22 pages.

[18] Totaro, B. The torsion index of the spin groups. Duke Math. J. 129, 2 (2005), 249-290.

[19] Vishik, A. On the Chow groups of quadratic Grassmannians. Doc. Math. 10 (2005), 111-130 (electronic).

[20] Voevodsky, V. Reduced power operations in motivic cohomology. Publ. Math. Inst. Hautes Études Sci., 98 (2003), 1-57.

[21] Yagita, N. The Gamma filtration for the Spin groups. arXiv:1811.08288 [math.KT], 17 Nov 2018, 27 pages.

Mathematical \& Statistical Sciences, University of Alberta, Edmonton, CANAdA

Email address: karpenko at ualberta.ca, web page: www.ualberta.ca/ ${ }^{k}$ arpenko 